\title{
A NECESSARY AND SUFFICIENT CONDITION OF NONRESONANCE FOR A SEMILINEAR NEUMANN PROBLEM
}

\author{
J. P. GOSSEZ AND P. OMARI
}

(Communicated by Barbara L. Keyfitz)

ABstract. We consider the Neumann problem

$$
\begin{cases}-\Delta u=g(u)+h(x) & \text { in } \Omega, \\ \partial u / \partial \nu=0 & \text { on bdry } \Omega .\end{cases}
$$

Assuming some growth restriction on the nonlinearity $g$, we prove that a necessary and sufficient condition for the existence of a solution for every given $h \in L^{\infty}(\Omega)$ is that $g$ be unbounded from above and from below on $\mathbb{R}$.

\section{INTRODUCTION} lem

This paper is concerned with the solvability of the following Neumann prob-

$$
\begin{cases}-\Delta u=g(u)+h(x) & \text { in } \Omega, \\ \partial u / \partial \nu=0 & \text { on bdry } \Omega .\end{cases}
$$

Here $\Omega$ is a bounded domain in $\mathbb{R}^{N}$, with $C^{1,1}$ boundary and unit exterior normal $\nu, g$ is continuous on $\mathbb{R}$, and $h$ is taken in $L^{\infty}(\Omega)$. By a solution of (1.1) we mean a function $u \in H^{1}(\Omega) \cap L^{\infty}(\Omega)$ satisfying

$$
\int_{\Omega} \nabla u \nabla v=\int_{\Omega} g(u) v+\int_{\Omega} h v
$$

for all $v \in H^{1}(\Omega)$. Of course, by the $L^{p}$ regularity theory, such a solution belongs to $W^{2, p}(\Omega)$ for all finite $p$.

We are interested in deriving conditions on the nonlinearity $g$ that guarantee nonresonance, i.e., the existence of a solution $u$ of (1.1) for every given $h$. Taking $v \equiv 1$ in (1.2), one immediately realizes that a necessary condition for nonresonance is that

$$
g \text { is unbounded from above and from below on } \mathbb{R} \text {. }
$$

This condition alone is not sufficient, as it is seen by taking for instance $g(s)=$ $\lambda s$, where $\lambda$ is a nonzero eigenvalue of the associated linear problem

$$
\begin{cases}-\Delta u=\lambda u & \text { in } \Omega, \\ \partial u / \partial \nu=0 & \text { on bdry } \Omega .\end{cases}
$$

Received by the editors July 27, 1990.

1980 Mathematics Subject Classification (1985 Revision). Primary 35J65, 35J25. 
This simple example suggests that some control should be imposed on the possible interaction of the nonlinearity $g$ with the higher part of the spectrum of (1.4). Denoting by $\lambda_{2}>0$ the second eigenvalue of (1.4), we will assume

$$
\limsup _{s \rightarrow \pm \infty} g(s) / s<\lambda_{2},
$$

or more generally

$$
\limsup _{s \rightarrow \pm \infty} g(s) / s \leq \lambda_{2} \text { and } \limsup _{\substack{s \rightarrow+\infty \\(\text { or } s \rightarrow-\infty)}} 2 G(s) / s^{2}<\lambda_{2},
$$

where $G(s)=\int_{0}^{s} g(t) d t$.

Theorem 1.1. Assume (1.5). Then a necessary and sufficient condition for (1.1) to be solvable for any $h \in L^{\infty}(\Omega)$ is that $g$ be unbounded from above and below on $\mathbb{R}$.

Several works have been devoted recently to questions of nonresonance for ordinary and partial differential equations. In particular, as far as (1.1) is concerned, the approach of [CoOl] can be adapted to derive nonresonance under (1.5) together with a similar condition at the right of the first eigenvalue $\lambda_{1}=0$ of (1.4):

$$
\liminf _{s \rightarrow \pm \infty} g(s) / s \geq \lambda_{1} \text { and } \liminf _{s \rightarrow \pm \infty} 2 G(s) / s^{2}>\lambda_{1} .
$$

Clearly, (1.6) implies (1.3). It also follows from [MWWi, Ma] that (1.3) implies nonresonance when $g$ is a nondecreasing function that has linear growth and satisfies (a weakened form of) (1.5). The proofs in [CoOl] and [MWWi, Ma] are based respectively on the Rabinowitz saddle point theorem and the dual least action principle of Clarke and Ekeland. Standard results on upper and lower solutions can also be used to derive nonresonance for (1.1) if $g$ is unbounded from above and below on $\mathbb{R}^{+}$, or from above and below on $\mathbb{R}^{-}$, or from above on $\mathbb{R}^{-}$and from below on $\mathbb{R}^{+}$. From this respect, Theorem 1.1 deals with the remaining case, where $g$ is unbounded from above on $\mathbb{R}^{+}$and from below on $\mathbb{R}^{-}$. A result analogous to Theorem 1.1 for periodic solutions of a class of second order ordinary differential equations was also obtained recently by the authors in $\left[\mathrm{GoO}_{2}\right]$.

The proof of Theorem 1.1 is based on the Leray-Schauder degree and involves the consideration of (constant) upper and lower solutions that may not be well ordered. It has some points in common with that in $\left[\mathrm{GoO}_{2}\right]$. In particular the open set that we eventually construct, in order to apply the invariance of the degree, is of the same type as the one introduced in $\left[\mathrm{GoO}_{2}\right]$. The main difference lies in the required estimate, which here provides a bound on the gradient in terms of the value of the function at any single point (cf. Claim 2 below). Assumption (1.5) with respect to $\lambda_{2}$ is exploited through its equivalence with a positive density condition (cf. the appendix in $\left.\left[\mathrm{GoO}_{2}\right]\right)$. Dealing with this positive density condition, in the case of a Neumann problem like here, requires a rather delicate lemma on the measure of inverse images given in [DFGo $\mathrm{DF}_{1}$.

It should be pointed out that a result analogous to Theorem 1.1 is not valid for the Dirichlet problem. Indeed, denoting by $\mu_{1}$ the first eigenvalue of $-\Delta$ on $H_{0}^{1}(\Omega)$, nonresonance for

$$
\begin{cases}-\Delta u=\mu_{1} u+g(u)+h(x) & \text { in } \Omega, \\ u=0 & \text { on bdry } \Omega,\end{cases}
$$


implies that (1.3) holds (multiply by the first eigenfunction and integrate). However an example was constructed recently in $[\mathrm{Nj}]$ of a continuous function $g$ that satisfies

$$
\operatorname{sgn}(s) g(s) \leq 0, \quad \liminf _{s \rightarrow \pm \infty} g(s) / s<0
$$

and for which $(1.7)$, with $N=1$, is not solvable for some smooth $h$. No general necessary and sufficient condition of nonresonance seems to be known for (1.7).

The proof of Theorem 1.1 is given in $\S 2$. In $\S 3$ we briefly consider a related result for jumping nonlinearities. In particular it is shown there that, when $g$ has linear growth, (1.5) can be replaced by a condition on $g(s) / s$ at $+\infty$ (or $-\infty)$ only (cf. Corollary 3.3).

\section{Proof of TheOREM 1.1}

Only the sufficient condition remains to be proved. Let us fix $p>N$. We start by recalling that an upper solution for (1.1) is defined as a function $\beta \in$ $W^{2, p}(\Omega)$ such that

$$
\left\{\begin{array}{l}
-\Delta \beta \geq g(\beta)+h(x) \text { a.e. in } \Omega, \\
\partial \beta / \partial \nu \geq 0 \text { on bdry } \Omega .
\end{array}\right.
$$

A lower solution $\alpha$ is defined by reversing these inequalities. The following lemma is well known, at least for $h$ continuous.

Lemma 2.1. Assume that (1.1) admits an upper solution $\beta$ and a lower solution $\alpha$, with $\alpha(x) \leq \beta(x)$ in $\Omega$. Then (1.1) has a soluition $u$, with $\alpha(x) \leq u(x) \leq$ $\beta(x)$ in $\Omega$.

The proof of Lemma 2.1 in the general case can be carried out by first taking a solution $u$ of

$$
\begin{cases}-\Delta u+u=f(x, u) & \text { in } \Omega, \\ \partial u / \partial \nu=0 & \text { on bdry } \Omega,\end{cases}
$$

where $f$ is defined through the following truncation:

$$
f(x, s)= \begin{cases}g(\beta(x))+\beta(x)+h(x) & \text { if } s>\beta(x), \\ g(s)+s+h(x) & \text { if } \alpha(x) \leq s \leq \beta(x), \\ g(\alpha(x))+\alpha(x)+h(x) & \text { if } s<\alpha(x) .\end{cases}
$$

By using $(u-\alpha)^{-}$and $(u-\beta)^{+}$as multipliers in the weak form of $(2.1)$, one then shows that $\alpha(x) \leq u(x) \leq \beta(x)$ in $\Omega$, which concludes the proof.

Lemma 2.1 allows us to take care of a few cases in the proof of Theorem 1.1. Indeed, if $g$ is unbounded from above and below on $\mathbb{R}^{+}$, then there exist $\alpha \leq \beta$ in $\mathbb{R}^{+}$such that $g(\alpha) \geq\|h\|_{\infty}$ and $g(\beta) \leq-\|h\|_{\infty}$. Taking the constant functions $\beta$ and $\alpha$ as upper and lower solutions, respectively, Lemma 2.1 applies and yields a solution of (1.1). The same situation occurs if $g$ is unbounded from above and below on $\mathbb{R}^{-}$, or unbounded from below on $\mathbb{R}^{+}$ and from above on $\mathbb{R}^{-}$.

It follows that we can assume from now on in the proof of Theorem 1.1 that for some constant $c_{1}$,

$$
\operatorname{sgn}(s) g(s) \geq c_{1},
$$


for every $s \in \mathbb{R}$. Moreover, we can find $A<0$ and $B>0$ such that

$$
g(A) \leq-\|h\|_{\infty} \quad \text { and } \quad g(B) \geq\|h\|_{\infty} .
$$

Observe that the constant functions $A$ and $B$ are upper and lower solutions respectively, but they are now ordered in the wrong way.

Let us fix a number $\vartheta$, with $0<\vartheta<\lambda_{2}$, and let us denote by $K$ the operator that sends $e(x)$ on the unique solution $u$ of

$$
\left\{\begin{array}{l}
-\Delta u=\vartheta u+e(x) \text { in } \Omega, \\
\partial u / \partial \nu=0 \text { on bdry } \Omega .
\end{array}\right.
$$

Then (1.1) is transformed into the fixed point equation

$$
u=K[-\vartheta u+g(u)+h(x)]
$$

in, say, $C^{1}(\operatorname{cl} \Omega)$, which will turn out to be a suitable functional setting. We will deal with the homotopy

$$
u=\mu K[-\vartheta u+g(u)=h(x)],
$$

where $\mu \in[0,1]$. Relation (2.5) corresponds to the problem

$$
\left\{\begin{array}{l}
-\Delta u=(1-\mu) \vartheta u+\mu[g(u)+h(x)] \text { in } \Omega, \\
\partial u / \partial \nu=0 \text { on bdry } \Omega .
\end{array}\right.
$$

Claim 1. There exists a constant $c_{2}$ (independent of $u$ and $\mu$ ) such that if $u$ solves (2.6), for some $\mu \in[0,1]$, then either $\max u \leq c_{2}$ or $\min u \geq-c_{2}$.

Proof of Claim 1. Assume by contradiction the existence of a sequence of numbers $\mu_{n} \in[0,1]$ and corresponding solutions $u_{n}$ of $(2.6)$ such that

$$
\max u_{n} \rightarrow+\infty \text { and } \min u_{n} \rightarrow-\infty .
$$

Put $v_{n}=u_{n} /\left\|u_{n}\right\|_{\infty}$. By the linear growth of $g$ (which follows from (1.5) and (2.2)) and the $L^{p}$ regularity theory, $\left\{v_{n}\right\}$ remains bounded in $W^{2, p}(\Omega)$ and so, for a subsequence, $v_{n} \rightarrow v$ weakly in $W^{2, p}(\Omega)$ and $\mu_{n} \rightarrow \mu \in[0,1]$. Clearly $\|v\|_{\infty}=1$. Also, for a further subsequence, $\left\{g\left(u_{n}\right) /\left\|u_{n}\right\|_{\infty}\right\}$ converges in $L^{\infty}(\Omega)$ with respect to the weak ${ }^{*}$ topology, and the limit function can be written as $m(x) v(x)$, with $0 \leq m(x) \leq \lambda_{2}$ a.e. in $\Omega$. This last point can be verified as in $[\mathrm{BDF}]$ or $\left[\mathrm{GoO}_{1}\right]$, using (2.2) and the first part of (1.5). It follows that $v$ satisfies

$$
\begin{cases}-\Delta v=p(x) v & \text { in } \Omega, \\ \partial v / \partial \nu=0 & \text { on bdry } \Omega,\end{cases}
$$

where $p(x)=(1-\mu) \vartheta+\mu m(x)$. We thus have $0 \leq p(x) \leq \lambda_{2}$ a.e. in $\Omega$. Three cases are now distinguished: (i) $p(x)=0$ a.e. in $\Omega$, (ii) $p(x)>0$ and $p(x)<\lambda_{2}$ on subsets of positive measure, (iii) $p(x)=\lambda_{2}$ a.e. in $\Omega$.

In case (i) $v$ is a nonzero constant and so $u_{n}=v_{n}\left\|u_{n}\right\|_{\infty} \rightarrow+\infty$ (or $-\infty$, depending on the sign of $v$ ) uniformly. This contradicts (2.7).

In case (ii) the contradiction comes from the following lemma, which can be proved by using Fourier series, as in [MaW] or the strict monotonicity dependence of eigenvalues in problems with weight (cf. $\left.\left[\mathrm{DFGo}_{2}\right]\right)$. 
Lemma 2.2. Let $\lambda_{i}<\lambda_{i+1}$ be two consecutive eigenvalues of (1.4). Assume that $p \in L^{\infty}(\Omega)$ satisfies $\lambda_{i} \leq p(x) \leq \lambda_{i+1}$ a.e. in $\Omega$, with $p(x)>\lambda_{i}$ and $p(x)<\lambda_{i+1}$ on subsets of positive measure. Then $u \equiv 0$ is the only solution of

$$
\begin{cases}-\Delta u=p(x) u & \text { in } \Omega, \\ \partial u / \partial \nu=0 & \text { on bdry } \Omega .\end{cases}
$$

In case (iii) $v$ is an eigenfunction corresponding to $\lambda_{2}, \mu=1$ and $m(x)=\lambda_{2}$ a.e. in $\Omega$. We also have in this case

$$
\int_{\Omega}\left(g\left(u_{n}\right) /\left\|u_{n}\right\|_{\infty}-\lambda_{2} v_{n}\right) v_{n} \rightarrow 0
$$

since $\left\{g\left(u_{n}\right) /\left\|u_{n}\right\|_{\infty}\right\}$ converges to $m v=\lambda_{2} v$. We will show that there exists $\eta>0$ and $\delta>0$ such that

$$
\mu_{N}\left\{x \in \Omega: u_{n}(x) \neq 0 \text { and } g\left(u_{n}(x)\right) / u_{n}(x) \leq \lambda_{2}-\eta\right\} \geq \delta,
$$

for all $n$ sufficiently large. Here $\mu_{N}$ denotes the Lebesgue measure in $\mathbb{R}^{N}$. Let us admit (2.10) for a moment and conclude the proof of Claim 1. Denote by $\chi_{n}$ the characteristic function of the set in (2.10). Decomposing the integral in (2.9) into the sum of an integral over the set in (2.10) and an integral over its complement, and using Fatou's lemma to deal with this second integral, one obtains

$$
\limsup _{n \rightarrow+\infty}\left(-\eta \int_{\Omega} v_{n}^{2} \chi_{n}\right) \geq 0 .
$$

So, for a subsequence, $\int_{\Omega} v_{n}^{2} \chi_{n} \rightarrow 0$, which implies $\int_{\Omega} v^{2} \chi_{n} \rightarrow 0$. A contradiction then easily follows from (2.10) and the fact that the eigenfunction $v$ does not vanish on a set of positive measure.

It remains to prove (2.10). Since $g$ has linear growth, it follows from the appendix in $\left[\mathrm{GoO}_{2}\right]$ that assumption (1.5) implies the existence of $\eta>0$ such that the set

$$
E=\left\{s \in \mathbb{R}: s \neq 0 \text { and } g(s) / s \leq \lambda_{2}-\eta\right\}
$$

has positive density at $+\infty$ (we are assuming here that the second half of (1.5) holds at $+\infty$; similar arguments should be used in the other case). We recall that the positive density of $E$ at $+\infty$ means that

$$
\liminf _{r \rightarrow+\infty} \mu_{1}(E \cap[0, r]) / \mu_{1}([0, r])>0 .
$$

We will need the following lemma on the measure of inverse images. Its proof, which uses the Steiner rearrangements, is given in [DFGo ${ }_{1}$.

Lemma 2.3. Let $\omega \subset \mathbb{R}^{N}$ be an open parallelepiped. Then there exists a constant $c=c(N, \omega)>0$ such that

$$
\mu_{N}\left(u^{-1}(B)\right) \geq c \mu_{1}(B)^{N} / \operatorname{Lip}(u)^{N},
$$

for any nonconstant Lipschitz function $u$ on $\omega$ and any Borelian set $B$ in the range of $u$.

To complete the proof of (2.10), let us take an open parallelepiped $\omega \subset \Omega$ such that $v$ changes sign on $\omega$. It follows that, given $0<\varepsilon<\sup _{\omega} v$, for all $n$ sufficiently large,

$$
\text { range of } v_{n} \text { on } \omega \supset\left[0, \sup _{\omega} v-\varepsilon\right] \text {, }
$$


and consequently

$$
\text { range of } u_{n} \text { on } \omega \supset\left[0,\left(\sup _{\omega} v-\varepsilon\right)\left\|u_{n}\right\|_{\infty}\right] \text {. }
$$

We apply Lemma 2.3 to the function $u_{n}$ on $\omega$ and to the Borelian set

$$
B_{n}=E \cap\left[0,\left(\sup _{\omega} v-\varepsilon\right)\left\|u_{n}\right\|_{\infty}\right],
$$

which, by (2.12), lies in the range of $u_{n}$ on $\omega$. This gives

$$
\mu_{N}\left\{x \in \Omega: u_{n}(x) \neq 0 \text { and } g\left(u_{n}(x)\right) / u_{n}(x) \leq \lambda_{2}-\eta\right\} \geq c \mu_{1}\left(B_{n}\right)^{N} / \operatorname{Lip}\left(u_{n}\right)^{N} \text {. }
$$

Since $v_{n}$ converges in $C^{1}(\operatorname{cl} \Omega), \operatorname{Lip}\left(v_{n}\right)$ remains bounded, and so

$$
\operatorname{Lip}\left(u_{n}\right) \leq c_{3}\left\|u_{n}\right\|_{\infty},
$$

for some constant $c_{3}$. Replacing in (2.13) and using (2.11) then yield (2.10). This completes the proof of Claim 1 .

Claim 2. There exists a constant $c_{4}$ (independent of $u, \mu$ and $x_{0}$ ) such that if $u$ solves $(2.6)$, for some $\mu \in[0,1]$, then

$$
\|\nabla u\|_{\infty} \leq c_{4}\left|u\left(x_{0}\right)\right|^{(p-1) / p}+c_{4},
$$

for all $x_{0} \in \operatorname{cl} \Omega$.

Proof of Claim 2. Let $u$ be a solution of $(2.6)$, for some $\mu \in[0,1]$. Setting

$$
\varphi(u, \mu)=(1-\mu) \vartheta u+\mu g(u),
$$

we derive from (2.6) that

$$
\left|\int_{\Omega} \varphi(u, u)\right| \leq c
$$

where, here and below, $c$ represents a constant independent of $u, \mu$, and $x_{0}$, but which may vary from inequality to inequality. By Claim 1 , we have $\max u \leq c_{2}$ or $\min u \geq-c_{2}$. Consider the first case (the second can be treated similarly). It follows from (2.14) that

$$
-\int_{[\varphi<0]} \varphi(u, \mu) \leq c+\int_{[\varphi>0] \cap[u \leq 0]} \varphi(u, \mu)+\int_{[\varphi>0] \cap[u>0]} \varphi(u, \mu) .
$$

We use (2.2) in the second integral and $\max u \leq c_{2}$ in the third integral to obtain

$$
-\int_{[\varphi<0]} \varphi(u, \mu) \leq c .
$$

Combining with (2.14) then yields

$$
\|\varphi(u, \mu)\|_{L^{\prime}} \leq c .
$$

This implies, by the linear growth of $g$,

$$
\|\varphi(u, \mu)\|_{L^{p}} \leq\|\varphi(u, \mu)\|_{L^{1}}^{1 / p}\|\varphi(u, \mu)\|_{\infty}^{(p-1) / p} \leq c\|u\|_{\infty}^{(p-1) / p}+c .
$$

Let us write $w=u-(1 / \operatorname{meas}(\Omega)) \int_{\Omega} u$. By the basic estimates of the $L^{p}$ regularity theory and the last inequality above, we have

$\|\nabla u\|_{\infty}=\|\nabla w\|_{\infty} \leq\|w\|_{C^{1}} \leq c\|w\|_{W^{2, p}} \leq c\left(\|\varphi(u, \mu)+h\|_{L^{p}}\right) \leq c\|u\|_{\infty}^{(p-1) / p}+c$ 
and consequently, for every $x_{0} \in \mathrm{cl} \Omega$,

$$
\|\nabla u\|_{\infty} \leq c\left\|u-u\left(x_{0}\right)\right\|_{\infty}^{(p-1) / p}+c\left|u\left(x_{0}\right)\right|^{(p-1) / p}+c .
$$

Now, by the $C^{1}$ character of bdry $\Omega$, there exists a constant $c_{5}$ such that, for any $x, x_{0} \in \operatorname{cl} \Omega$, there is a smooth curve $\gamma$, with range contained in $\operatorname{cl} \Omega$, joining $x_{0}$ to $x$ and of length $\leq c_{5}$. This implies

$$
\left|u(x)-u\left(x_{0}\right)\right|=\left|\int_{[0,1]}\left\langle\nabla u(\gamma(t)), \gamma^{\prime}(t)\right\rangle d t\right| \leq c_{5}\|\nabla u\|_{\infty} .
$$

Using this inequality in (2.15), one easily gets the estimate of Claim 2 .

We are now ready to conclude the proof of Theorem 1.1. Define

$$
\begin{aligned}
& \mathscr{O}=\left\{u \in C^{1}(\operatorname{cl} \Omega): A<u\left(x_{0}\right)<B,\right. \\
& \text { for at least one } \left.x_{0} \in \operatorname{cl} \Omega, \text { and }\|\nabla u\|_{\infty}<C\right\},
\end{aligned}
$$

where $C=c_{4} \max \{-A, B\}^{(p-1) / p}+c_{4}+1$. Clearly, $\mathscr{O}$ is a bounded open subset of $C^{1}(\mathrm{cl} \Omega)$ which contains 0 . By the Leray-Schauder degree theory, (2.4) will be solvable in $\mathrm{cl} \mathscr{O}$ if no solution of (2.5), with $0 \leq \mu<1$, belongs to the boundary of $\mathscr{O}$. Assume by contradiction that there exists such a solution $u$ in bdry $\mathscr{O}$. Then, by Claim 2 and the choice of $C$ above, we conclude that $\max u=A$ or $\min u=B$. Consider the first case (the second can be treated similarly). Take $x_{1} \in \operatorname{cl} \Omega$, with $u\left(x_{1}\right)=\max u$. We distinguish two cases: either $x_{1} \in \Omega$ or $x_{1} \in$ bdry $\Omega$.

In the first case we start by observing that, by (2.3),

$$
(1-\mu) \vartheta A+\mu[g(A)+h(x)] \leq-\varepsilon,
$$

for some $\varepsilon>0$ and a.e. $x \in \Omega$. Consequently, by continuity,

$$
-\Delta(u-A)(x)=(1-\mu) \vartheta u(x)+\mu[g(u(x))+h(x)]<0,
$$

a.e. in some ball $B\left(x_{1}, r\right) \subset \Omega$. The strong maximum principle (cf. [GiTr, Theorem 8.19]) then implies that $u-A$ is constant on $B\left(x_{1}, r\right)$ which contradicts (2.16).

In the second case we derive as above that, for some ball $B\left(x_{1}, r\right)$,

$$
-\Delta(u-A)(x)<0,
$$

a.e. in $B\left(x_{1}, r\right) \cap \Omega$. By the $C^{1,1}$ character of bdry $\Omega$, the interior sphere condition is satisfied at $x_{1}$ and so the boundary form of the strong maximum principle (cf. [DF, Proposition 1.16]) implies that either $u-A$ is constant on $B\left(x_{1}, r\right) \cap \Omega$ or $\partial u / \partial \nu\left(x_{1}\right)>0$. In any case we get a contradiction. This completes the proof of Theorem 1.1.

Remark 2.4. The result of Theorem 1.1 easily extends to the problem

$$
\left\{\begin{array}{l}
\mathfrak{L} u=g(u)+h(x, u, \nabla u) \text { in } \Omega, \\
\partial u / \partial \nu_{\mathfrak{L}}=0 \text { on bdry } \Omega,
\end{array}\right.
$$

where $\mathfrak{L}$ is a uniformly strongly elliptic operator of the form

$$
\mathfrak{L}=-\sum_{i, j=1, \ldots, N} \partial / \partial x_{i}\left(a_{i j} \partial / \partial x_{j}\right)
$$


with Lipschitz coefficients $a_{i j}=a_{j i}, \partial / \partial \nu_{\mathfrak{L}}$ denotes the associated conormal derivative and $h$ is a bounded Carathéodory function. Observe that the unique continuation property, which is needed in the proof of Lemma 2.2 as well as in the application of (2.10) to the proof of Claim 1, now follows from [GL]. Observe also that our proof of Lemma 2.1 does not extend to the gradient dependent case. One should here restrict oneself to constant upper and lower solutions (which is all we need) and adapt the proof of Lemma 3.1 in $\left[\mathrm{GoO}_{2}\right]$, using the maximum principle in Sobolev spaces. (We refer to [Ad] for a recent study of the method of upper and lower solutions for Carathéodory type equations.)

Remark 2.5. Not well-ordered upper and lower solutions were already considered in [AAmM], but by totally different techniques and for bounded nonlinearities. The approach in [AAmM] can be extended to deal with some unbounded nonlinearities, as we shall show elsewhere. In this way one obtains a completely different proof of Theorem 1.1 (under, however, a slightly stronger condition than (1.5)). Results in this direction, in the context of periodic solutions for a class of ordinary differential equations, have been obtained by one of the authors in [O].

Remark 2.6. From the proof of Theorem 1.1 we can also derive sometimes the existence of multiple solutions. This is due to the fact that, in establishing the existence of a solution, our proof also provides some information about its location (cf. Lemma 2.1 and the definition of the set $\mathscr{O}$ above). In this way we can recover in particular some multiplicity results obtained in [DT].

\section{A RELATED RESULT FOR JUMPING NONLINEARITIES}

Assumption (1.5) imposes a restriction on the asymptotic behaviour of the nonlinearity $g$ with respect to the spectrum of (1.4). In this section we consider another type of restriction which somehow involves the Fučik spectrum of the associated linear operator.

The following lemma should be compared with Lemma 2.2. Its proof, by contradiction, can be easily adapted from that of corresponding lemmas in [Ah, INW].

Lemma 3.1. For any given $q_{-}>0$ there exists $q_{+}>0$ such that, if $p_{ \pm} \in L^{\infty}(\Omega)$ satisfy $0 \leq p_{-}(x) \leq q_{-}$and $0 \leq p_{+}(x) \leq q_{+}$a.e. in $\Omega$, with $p_{-}(x)>0$ and $p_{+}(x)>0$ on subsets of positive measure, then $u \equiv 0$ is the only solution of

$$
\left\{\begin{array}{l}
-\Delta u=p_{+}(x) u^{+}-p_{-}(x) u^{-} \text {in } \Omega, \\
\partial u / \partial \nu=0 \text { on bdry } \Omega .
\end{array}\right.
$$

Proposition 3.2. Let $q_{-}$and $q_{+}$be as in the above lemma. Assume

$$
\limsup _{s \rightarrow \pm \infty} g(s) / s<q_{ \pm} .
$$

Then the conclusion of Theorem 1.1 holds.

The proof of Proposition 3.2 proceeds along similar lines as that of Theorem 1.1. In the definition of the homotopy (cf. (2.5)), one takes $0<v<$ 
$\min \left\{q_{-}, q_{+}\right\}$. The limiting equation (2.8) in the proof of Claim 1 is now replaced by an equation of the form

$$
\left\{\begin{array}{l}
-\Delta v=p_{+}(x) v^{+}-p_{-}(x) v^{-} \text {in } \Omega \\
\partial v / \partial \nu=0 \text { on bdry } \Omega
\end{array}\right.
$$

to which Lemma 3.1 applies. No argument involving density is needed anymore. Claim 2 and the rest of the proof of Theorem 1.1 are unchanged.

Corollary 3.3. Assume

$$
\limsup _{s \rightarrow-\infty} g(s) / s<+\infty \text { and } \limsup _{s \rightarrow+\infty} g(s) / s \leq 0 \text {. }
$$

Then the conclusion of Theorem 1.1 holds.

Remark 3.4. The role of $q_{-}$and $q_{+}$in Lemma 3.1, as well as the limits in Corollary 3.3, can of course be interchanged.

Remark 3.5. More precise results involving the Fučik spectrum can be obtained in the case of ordinary differential equations (cf. $\left[\mathrm{GoO}_{2}\right]$ ).

\section{REFERENCES}

[Ad] A. Adje, Sur et sous solutions dans les équations différentielles discontinues avec conditions aux limites non linéaires, Thèse de doctorat, Univ. Louvain-la-Neuve, 1987.

[Ah] S. Ahmad, Nonselfadjoint resonance problems with unbounded perturbations, Nonlinear Anal. 10 (1986), 147-156.

[AAmM] H. Amann, A. Ambrosetti, and G. Mancini, Elliptic equations with noninvertible Fredholm linear part and bounded nonlinearities, Math. Z. 158 (1978), 179-194.

[BDF] H. Berestycki and D. G. De Figueiredo, Double resonance in semilinear elliptic problems, Comm. Partial Differential Equations 6 (1981), 91-120.

[CoOl] D. G. Costa and A. S. Oliveira, Existence of solutions for a class of semilinear elliptic problems at double resonance, Bol. Soc. Brasil. Mat. 19 (1988), 21-37.

[DF] D. G. De Figueiredo, Positive solutions of semilinear elliptic problems, Lecture Notes in Math., vol. 957, Springer-Verlag, Berlin, 1982, pp. 34-87.

$\left[\mathrm{DFGo}_{1}\right]$ D. G. De Figueiredo and J. P. Gossez, Nonresonance below the first eigenvalue for a semilinear elliptic problems, Math. Ann. 281 (1988), 589-610.

$\left[\mathrm{DFGo}_{2}\right] \ldots$ Strict monotonicity of eigenvalues and the unique continuation property (to appear).

[DT] P. Drábek and S. A. Tersian, Characterization of the range of Neumann problem for semilinear elliptic euations, Nonlinear Anal. 11 (1987), 733-739.

[GL] N. Garofalo and F. H. Lin, Unique continuation property for elliptic operators: a geometricvariational approach, Comm. Pure Appl. Math. 40 (1987), 347-366.

[GiTr] D. Gilbarg and N. S. Trudinger, Elliptic partial differential equations of the second order, Springer-Verlag, Berlin, 1983.

$\left[\mathrm{GoO}_{1}\right]$ J. P. Gossez and P. Omari, Nonresonance with respect to the Fučik spectrum for periodic solutions of second order ordinary differential equations, Nonlinear Anal. 14 (1990), 10791104.

$\left[\mathrm{GoO}_{2}\right] \ldots$, Periodic solutions of a second order ordinary differential equation: a necessary and sufficient condition for nonresonance, J. Diff. Equat. (to appear).

[INW] R. Iannacci, M. N. Nkashama, and J. R. Ward, Jr., Nonlinear second order elliptic partial differential equations at resonance, Trans. Amer. Math. Soc. 311 (1989), 711-726.

[Ma] J. Mawhin, A Neumann boundary value problem with jumping monotone nonlinearity, Delft Progress Report 10 (1985), 44-52.

[MaW] J. Mawhin and J. R. Ward, Jr., Nonresonance and existence for nonlinear elliptic boundary value problems, Nonlinear Anal. 6 (1981), 677-684. 
[MWWi] J. Mawhin, J. R. Ward, Jr., and M. Willem, Variational methods and semi-linear elliptic equations, Arch. Rational Mech. Anal. 95 (1986), 269-277.

[Ni] F. I. Njoku, Some remarks on the solvability of the nonlinear two-point boundary value problems, preprint.

[O] P. Omari, Non-ordered lower and upper solutions and solvability of the periodic problem for the Liénard and the Rayleigh equations, preprint.

Départment de Mathématique, Campus Plaine, C.P. 214, Université libre de Bruxelles, 1050 BruXelles, BelgiQue

Dipartimento di Scienze Matematiche, Università di Trieste, Piazzale Europa 1, 34127 TRIESTE, ITALIA 